\title{
Successful recruitment and retention of diverse participants in a genomics clinical trial: a good invitation to a great party
}

\author{
Carol R. Horowitz, MD, MPH ${ }^{1}{ }^{1}$, Tatiana Sabin, $\mathrm{BA}^{1}$, Michelle Ramos, $\mathrm{MPH}^{1}$, \\ Lynne D. Richardson, MD ${ }^{1,2}$, Diane Hauser, MPA ${ }^{3}$, Mimsie Robinson, MA ${ }^{4}$ and Kezhen Fei, $\mathrm{MS}^{1}$
}

Purpose: African ancestry (AA) individuals are inadequately included in translational genomics research, limiting generalizability of findings and benefits of genomic discoveries for populations already facing disproportionately poor health outcomes. We aimed to determine the impact of stakeholder-engaged strategies on recruitment and retention of AA adult patients into a clinical trial testing them for renal risk variants nearly exclusive to AAs.

Methods: Our academic-clinical-community team developed ten key strategies that recognize AAs' barriers and facilitators for participation. Using electronic health records (EHRs), we identified potentially eligible patients. Recruiters reached out through letters, phone calls, and at medical visits.

Results: Of 5481 AA patients reached, 51\% were ineligible, 37\% enrolled, $4 \%$ declined, $7 \%$ were undecided when enrollment finished. We retained $93 \%$ at 3 -month and $88 \%$ at 12 -month follow-up. Those enrolled are more likely female, seen at community sites, and reached through active strategies, than those who declined. Those retained are more likely female, health-literate, and older. While many patients have low income, low clinician trust, and perceive racism in health care, none of these attributes correlate with retention.

Conclusion: With robust stakeholder engagement, recruiters from patients' communities, and active approaches, we successfully recruited and retained AA patients into a genomic clinical trial.

Genetics in Medicine (2019) 21:2364-2370; https://doi.org/10.1038/s41436019-0498-x

Keywords: African ancestry; race; recruitment; retention; genomics

\section{INTRODUCTION}

Research with generalizable results should include sociodemographically diverse participants, yet researchers often struggle to recruit and retain diverse participants in clinical trials. Barriers to participation among racial-ethnic minority and low-income individuals include distrust, concerns about time required, privacy, past research misconduct, and experiences with implicit bias, discrimination, and racism. ${ }^{1,2}$ Researchers encounter recruitment challenges due to participants' linguistic, literacy, and logistic barriers. ${ }^{3,4}$ Potential benefits for patients to participate in research may not sufficiently outweigh concerns. ${ }^{5}$ It is important to intentionally, actively, and thoughtfully design studies with inclusivity in mind. ${ }^{6}$

Recruitment difficulties may be magnified in genomic research, where it is critical to include diverse populations to facilitate equal access to scientific breakthroughs, address biases and inaccuracies of findings that can occur when research is conducted solely in nonminority groups, and to use findings to guide health practices, policies, and address health disparities. ${ }^{7,8}$ Known barriers to genomic testing and research participation in genomic studies such as insurance coverage, mistrust, complex trial or consent processes, fear of bad news, time required, and distance are amplified in minority populations. ${ }^{4,5,9-11}$ African Americans have a lower predisposition toward genomic studies and are more likely to report them as harmful to society. They also have additional concerns about privacy and whether genomic information will be used against them by the government, health-care system, or law enforcement-issues that disproportionately affect them and are seen through a lens of historic discrimination. ${ }^{6,12,13}$

Little is known about how to offer genomic testing in primary care: even less is known about the impact of genomic testing for chronic disease risk, particularly among racial-ethnic minority patients who have the highest prevalence and poorest outcomes from many common illnesses due to clinical, socioeconomic, and environmental

${ }^{1}$ Center for Health Equity and Community Engaged Research and Department of Population Health Science and Policy, Icahn School of Medicine at Mount Sinai, New York, NY, USA; ${ }^{2}$ Department of Emergency Medicine, Icahn School of Medicine at Mount Sinai, New York, NY, USA; ${ }^{3}$ The Institute for Family Health, New York, NY, USA; ${ }^{4}$ Bethel Gospel Assembly, New York, NY, USA. Correspondence: Carol R. Horowitz (carol.horowitz@mssm.edu) 
factors, yet are least likely to be enrolled in genomic studies. $^{14,15}$

Our team of researchers, clinicians, patients, and advocates has had success employing patient-centered, culturally and linguistically appropriate techniques to recruit and retain diverse participants in chronic disease prevention and control clinical trials. ${ }^{16-18}$ We aimed to use these experiences to recruit patients into the Genetic testing to Understand and Address Renal Disease Disparities (GUARDD) Trial, part of the National Institutes of Health's first genomic medicine implementation network. ${ }^{19}$ GUARDD randomized adults with self-reported African ancestry (AA), including those who self-identify as Black, African American, Afro-Latino, Afro-Caribbean, or African, to receive immediate or delayed testing for renal risk variants in the APOL1 genes, which are found nearly exclusively in AAs and significantly increase their risk of kidney failure. ${ }^{20}$ Here, we assess our success engaging, recruiting, and retaining AA patients in this study.

\section{MATERIALS AND METHODS}

During initial visits, recruiters consented patients, collected clinical measures, and verbally administered a survey to assess attitudes and behaviors around hypertension, kidney disease, and genomic testing. ${ }^{21}$ Recruiters were trained by a senior genetic counselor to consent patients and disclose APOL1 genetic test results, and conducted 3- and 12-month follow-up study visits within a window of two weeks before to one month after the due date.

We developed and implemented recruitment and retention protocols approved by the Icahn School of Medicine and Institute for Family Health Institutional Review Boards (IRBs) using procedures to contextualize our results and allow others to adopt promising practices:

1. Stakeholder engagement. A genomics board of researchers, patients, advocates, primary care and genomic medicine physicians, and genetic counselors codeveloped the study design, materials, and recruitment and retention strategies and helped brainstorm solutions to challenges faced during recruitment. ${ }^{22}$

2. Formative work. We conducted in-depth interviews with patients to introduce the consent form, conduct testing, and disclose results. We used this information to inform our protocol. ${ }^{23}$ For example, patients said receiving negative test results by phone and having briefer pretest counseling would reduce study burden and increase their interest in taking part.

3. Clinician buy-in. A study clinician met with primary care providers (PCPs) at each site during one of their regularly scheduled meetings, explained the purpose of the study, answered their questions, asked them to sign or agree to send a letter from their practice (which we would send to their patients), and provided them with study contact cards to refer eligible patients.

4. Study materials and stipends. We developed low-literacy consent forms, letters, call scripts, and flyers to resonate with our patient population, keeping in mind reasons highlighted in the literature for hesitancy and difficulty participating in research, and "earned mistrust" in the research enterprise. ${ }^{24}$ When a local IRB was hesitant to adopt changes to the consent form, stakeholders met with them and came to consensus on language that was understandable and met legal and regulatory requirements. The Stakeholder Board chose patient stipends for time spent on travel and research-specific visits that they viewed as respectful but not coercive ( $\$ 40$ per visit via a choice of gift cards to popular local stores).

5. Recruiter training and oversight. We trained recruiters to use procedures proven effective in recruiting minority patients, ${ }^{18-20}$ provided feedback in mock study visits, and piloted and revised screening questions and surveys to minimize patient burden. At biweekly meetings, we used REDCap-generated reports to track recruitment by recruiter and site, discuss challenges, and brainstorm approaches to handle difficult situations. ${ }^{25}$ Recruiters were also sources of support and guidance for each other in identifying challenges and solutions based on their experiences with patients.

6. Efficient patient identification in a variety of settings. The 15 primary care study sites included community (federally qualified health centers and small neighborhood practices) and academic practices. We identified potentially eligible patients via EHRs, with specific visit criteria ( $\geq 1$ PCP visit at a study site in the past year), demographic criteria (Black, 18-70 years old) and clinical criteria (EHR documentation of hypertension and/or taking antihypertensive medications and/or two or more blood pressure readings above 140/90 $\geq$ 6 months apart; excluding those with diagnoses of diabetes, pregnancy, kidney disease, or cognitive impairment). ${ }^{21}$

7. Flexible, targeted outreach and scheduling. For baseline and follow-up visits, recruiters called patients, including at least one evening and one weekend call, and scheduled recruitment and follow-up visits at a convenient time including evenings, weekends, and on the same day as a doctor's appointment. Recruiters sent reminder letters, emails, or texts. If recruiters could not reach patients, we identified upcoming appointments, so recruiters could intercept them to ascertain their interest or schedule visits when they were on site. Our REDCap database included all potentially eligible patients and calendars with upcoming appointments and follow-up due dates of participants.

8. Study branding. We mailed patient letters in bright blue envelopes; our logo was on all patient-facing materials and recruiters wore bright blue lab coats. Each recruiter was assigned to a few specific sites, where they got to know clinicians and staff, and would ask them to contact the "blue-coat" to refer patients. We placed flyers and posters in study sites and advertised in clinical newsletters. 
9. Relationship-centered recruitment and retention. We assigned recruiters (from similar neighborhoods or racial/ethnic backgrounds as patients) to specific patients with whom they took time to get to know and build rapport during and between visits. As recruiters spent much of their time in and around the study practices, they ran into patients frequently. The Genomics Board recommended we contact participants between 3- and 12-month follow-ups, so we sent birthday and holiday cards, a 6-month postcard reminding them about the study, and placed a scripted 9-month call.

10. Collect data for impact of strategies. To compare those enrolled versus declined, we asked patients who declined one open-ended question, and supplemented this with REDCap data on how we approached patients (e.g., phone call vs. intercept) and EHR demographic data. To compare those eligible versus ineligible, we collected reasons from patients and supplementary EHR review. For retention, we utilized EHR and baseline survey data to compare those retained versus lost to follow-up. Chisquare statistics compared group differences for categorical variables and $t$ tests assessed continuous variables, with significance level set at 0.05 .

\section{RESULTS}

In two years, we completed our planned enrollment of 2052 patients. We identified 7959 eligible adults through EHRs. We did not complete eligibility screening on 2478 (31\%): 71 returned refusal letters, and we stopped contacting 2407 when we met our prespecified enrollment goal. All PCPs gave us permission to approach all their patients for the study; none withdrew permission. Of the 5481 patients reached, $51 \%$ were ineligible, $37 \%$ enrolled, $4 \%$ declined, and $7 \%$ were still deciding whether to participate when we completed enrollment.

Several factors explained the high rate of ineligible patients. Due to limitations of EHR identification, many patients stated they were erroneously labeled as Black or African American (13\%), having hypertension (32\%), or stated they had kidney disease or diabetes (12\%). Others (31\%) were no longer patients at any participating clinical site so we could not return results to their PCPs through EPIC, as specified in our protocol. Some were institutionalized or terminally ill (8\%); a few (4\%) did not speak English. In comparing the limited data we could obtain for ineligible to eligible patients (Table 1 and 2 ), there were no statistically significant differences in mean age, but more eligibles were female, from community (vs. academic) clinical sites, and had Medicare (vs. commercial, Medicaid, or no insurance) (all $p<0.05$ ).

Patients declined to participate because they were too busy/ had competing demands (22\%), were not interested in research in general (35\%), not interested in genomics research $(5 \%)$, too ill $(2 \%)$, had other reasons $(7 \%)$, or gave no reason (29\%). We compared those enrolled with those who declined
Table 1 Keys to recruitment and retention in translational genomic studies

\begin{tabular}{|c|c|}
\hline Measure & Rationale \\
\hline 1. Stakeholder engagement & $\begin{array}{l}\text { Develop engaging, culturally and } \\
\text { literacy-appropriate strategies and } \\
\text { materials; address problems that arise }\end{array}$ \\
\hline 2. Formative research & $\begin{array}{l}\text { Uncover and pilot approaches and } \\
\text { strategies }\end{array}$ \\
\hline 3. Clinician buy-in & Facilitate recruitment \\
\hline $\begin{array}{l}\text { 4. Study materials and } \\
\text { stipends }\end{array}$ & $\begin{array}{l}\text { Resonate with, educate, and respectfully } \\
\text { but not coercively introduce study }\end{array}$ \\
\hline $\begin{array}{l}\text { 5. Recruiter training, } \\
\text { recruitment oversight }\end{array}$ & $\begin{array}{l}\text { Rapidly identify challenges and solve } \\
\text { using a team approach }\end{array}$ \\
\hline $\begin{array}{l}\text { 6. Efficient patient } \\
\text { identification in a variety of } \\
\text { settings }\end{array}$ & $\begin{array}{l}\text { Identify broad and diverse patient } \\
\text { phenotype }\end{array}$ \\
\hline $\begin{array}{l}\text { 7. Flexible, targeted outreach, } \\
\text { scheduling }\end{array}$ & $\begin{array}{l}\text { Maximize ability for individuals to } \\
\text { participate and show respect for them }\end{array}$ \\
\hline 8. Study branding & $\begin{array}{l}\text { Build study buzz and recognition, } \\
\text { facilitate patient outreach to study team }\end{array}$ \\
\hline $\begin{array}{l}\text { 9. Relationship-centered } \\
\text { recruitment and retention }\end{array}$ & $\begin{array}{l}\text { Build community of researchers, } \\
\text { recruiters, patients, clinicians whose } \\
\text { needs and input are valued and } \\
\text { incorporated }\end{array}$ \\
\hline $\begin{array}{l}\text { 10. Collect data on impact of } \\
\text { strategies }\end{array}$ & $\begin{array}{l}\text { Assess success, plan for future studies, } \\
\text { build evidence base }\end{array}$ \\
\hline
\end{tabular}

Table 2 Patients approached: ineligible versus eligible

\begin{tabular}{llll}
$N=5481$ & $\begin{array}{l}\text { Ineligible } \\
(N=2783)\end{array}$ & $\begin{array}{l}\text { Eligible } \\
(N=2698)\end{array}$ & $p$ \\
\hline Age, mean, years & 52.6 & 52.5 & 0.8 \\
\hline Female & $60 \%$ & $65 \%$ & 0.0002 \\
\hline Insurance & & & $<0.0001$ \\
\hline Private & $41 \%$ & $45 \%$ & \\
Medicaid & $27 \%$ & $40 \%$ & \\
Medicare & $20 \%$ & $9 \%$ & \\
None & $3 \%$ & $2 \%$ & 0.02 \\
\hline Site type: community & $56 \%$ & $59 \%$ & \\
\hline
\end{tabular}

or who had not yet decided to participate when enrollment closed, conservatively estimating that many undecided patients may have ultimately declined. Those who enrolled were more likely female, to see PCPs at community sites, to have Medicaid, be reached through in-person clinic intercepts and clinician referrals (vs. phone calls), and to proactively reach us (call in response to letters, flyers, and posters) (all $p$ $<0.05$; Table 3 ).

We enrolled patients through several outreach strategies: $28 \%$ contacted us in response to our outreach letter, $23 \%$ agreed during telephone calls, $26 \%$ were intercepted at a clinician visit, $7 \%$ were referred by a PCP, and $13 \%$ responded 
Table 3 Patients eligible: enrolled versus declined/ undecided

\begin{tabular}{llll}
$N=2698$ & $\begin{array}{l}\text { Declined/undecided } \\
(N=646)\end{array}$ & $\begin{array}{l}\text { Enrolled } \\
(N=2052)\end{array}$ & $p$ \\
\hline Age, mean years & 51.2 & 52.9 & 0.0004 \\
\hline Female & $60 \%$ & $66 \%$ & 0.004 \\
\hline $\begin{array}{l}\text { Insurance } \\
\text { Private }\end{array}$ & $54 \%$ & $42 \%$ & $<0.0001$ \\
\hline Medicaid & $21 \%$ & $45 \%$ & \\
\hline Medicare & $17 \%$ & $7 \%$ & \\
\hline None & $1 \%$ & $3 \%$ & \\
\hline Site type: community & $55 \%$ & $60 \%$ & 0.02 \\
\hline Recruitment method & & & $<0.0001$ \\
\hline Letter & $24 \%$ & $28 \%$ & \\
\hline Telephone call & $54 \%$ & $23 \%$ & \\
\hline Clinic intercept & $19 \%$ & $26 \%$ & \\
\hline Clinician referral & $2 \%$ & $7 \%$ & \\
\hline Flyer, poster & $2 \%$ & $17 \%$ & \\
\hline
\end{tabular}

to flyers, newsletters, or posters. All enrolled individuals were of self-reported African ancestry, two-thirds were female (the same proportion as in our initial EHR data pull); a majority were first-time research participants, from community sites, and were low income (Table 4). Less than half reported trust in clinicians, more than three-quarters reported racism in health care, and more than a quarter reported inadequate social support. Only one-fourth reported having excellent or very good general health.

We had good retention at 3 months (93\%) and 12 months (88\%). At 3 months, those lost to follow-up were more likely male and first-time research participants, and less likely to be health-literate. There were no differences between the groups in other attributes (Table 4). At 12 months, males were still less likely to follow up, as were those younger and from community sites (all $p<0.05$ ); there were no other significant differences.

\section{DISCUSSION}

In the GUARDD trial, our diverse community-clinicalacademic-partnership aimed to recruit African ancestry patients with hypertension into a clinical trial to determine the health, health-care, and psychobehavioral impacts of testing them for genomic risk for kidney failure. There is limited involvement of racial-ethnic minority groups in genomic research, particularly in genomic medicine interventions, and we developed and implemented ten key strategies to maximize enrollment of a diversity of AA patients in the study (Table 1). Our population had what could be considered significant barriers to participating in research, including medical mistrust, perceived racism, depressive symptoms, anxiety, and relatively low health literacy and self-rated health. Yet, in two years, we successfully recruited over 2000 patients at academic, community, and safety net practices; only $4 \%$ declined and $7 \%$ were undecided when we completed enrollment. We retained $93 \%$ at 3 months and $88 \%$ at 12 months.

To maximize engagement, it is important to carefully design recruitment strategies with awareness and acknowledgement of patient needs and preferences. ${ }^{25,26} \mathrm{We}$ considered reasons minority individuals may not be interested in, or have difficulty joining, a genomics study, including mistrust in research and in genomic testing, competing demands, difficulties enrolling urban individuals in trials, disinterest in genomic testing for chronic disease risk (vs. for diagnosis or treatment), and concerns about eugenics. ${ }^{27-29}$ We attribute our low refusal and high retention rates to robust stakeholder engagement, patient-centered approaches, and development of outreach strategies with potential barriers and facilitators in mind. ${ }^{30}$

We view our success employing the strategies in Table $\mathbf{1}$ for recruitment and retention as "a good invitation to a great party." The good invitation signifies that we designed every outreach strategy, piece of written material, and conversation with-and for-our target population so patients and PCPs might feel that their colleagues and neighbors shaped the study with them specifically in mind. People from patients' communities reached out to them with materials developed and approved by their neighbors (racial concordance does enhance recruitment success) ${ }^{9}$ and study PCPs introduced and updated site PCPs about GUARDD.

Consistent with the literature, recruitment strategies that garnered the highest response rates were the most proactive and targeted methods (such as clinic intercepts and telephone calls). Such approaches are more time-consuming. ${ }^{8} \mathrm{We}$ cannot prove that incentives increased participation rates; literature about their effectiveness is inconclusive and some point out ethical concerns about incentives. ${ }^{3,31}$ We did not randomize people to receive or not receive stipends, as we feel ethically compelled to provide stipends when participants come for study visits that are not related to clinical care and may not provide clinical benefit.

Our strategies minimized common logistic barriers such as time and travel. We had 15 sites where people could join the study, including where they usually receive care, and we had evening and weekend hours. Including a variety of recruitment sites, particularly community-based clinics, can help address enrollment barriers. Community sites tend to be more diverse sources for recruitment and provide patients with a familiar and convenient location for visits, with staff they trust. $^{8}$

Those who declined were more likely younger and male. Women are more frequently excluded from clinical trials than men $^{32}$ and some studies show women are more difficult to recruit than men. ${ }^{33}$ We encountered the opposite challenge. The majority of patients who declined stated that they were not interested in participating in research, but only a very small percentage specified that they were not interested in genomic research specifically. This goes against our initial assumptions that patients might be opposed to genomic research in particular. 
Table 4 Patient characteristics by follow-up status at 3 months and 12 months

\begin{tabular}{|c|c|c|c|c|c|c|c|}
\hline & \multirow{2}{*}{$\begin{array}{l}\text { Baseline }(n=2052) \\
N=2052\end{array}$} & \multicolumn{2}{|c|}{ 3-month follow-up } & \multirow[t]{2}{*}{$p$} & \multicolumn{2}{|c|}{ 12-month follow-up } & \multirow[t]{2}{*}{$p$} \\
\hline & & $\begin{array}{l}\% \text { Not complete } \\
N=134\end{array}$ & $\begin{array}{l}\% \text { Complete } \\
N=1918\end{array}$ & & $\begin{array}{l}\% \text { Not complete } \\
N=244\end{array}$ & $\begin{array}{l}\% \text { Complete } \\
N=1808\end{array}$ & \\
\hline Female & $66 \%$ & $54 \%$ & $67 \%$ & 0.002 & $60 \%$ & $67 \%$ & 0.03 \\
\hline Insurance & & & & 0.94 & & & 0.23 \\
\hline Private & $42 \%$ & $43 \%$ & $42 \%$ & & $45 \%$ & $42 \%$ & \\
\hline Medicare & $7 \%$ & $6 \%$ & $7 \%$ & & $5 \%$ & $7 \%$ & \\
\hline None & $3 \%$ & $2 \%$ & $3 \%$ & & $2 \%$ & $3 \%$ & \\
\hline Live with partner & $30 \%$ & $36 \%$ & $30 \%$ & 0.12 & $35 \%$ & $29 \%$ & 0.09 \\
\hline Income $<\$ 30,000 /$ year & $56 \%$ & $58 \%$ & $56 \%$ & 0.68 & 56 & $56 \%$ & 0.96 \\
\hline Education $\leq \mathrm{HS}$ & $43 \%$ & $42 \%$ & $43 \%$ & 0.87 & $45 \%$ & $42 \%$ & 0.37 \\
\hline Perceive racism in medical care & $80 \%$ & $85 \%$ & $80 \%$ & 0.19 & $76 \%$ & $81 \%$ & 0.09 \\
\hline Have social support & $74 \%$ & $72 \%$ & $74 \%$ & 0.62 & $77 \%$ & $73 \%$ & 0.19 \\
\hline Community site & $60 \%$ & $61 \%$ & $60 \%$ & 0.74 & $68 \%$ & $59 \%$ & 0.008 \\
\hline Uncontrolled BP & $45 \%$ & $53 \%$ & $45 \%$ & 0.06 & $45 \%$ & $45 \%$ & 0.9 \\
\hline$\geq 1$ Comorbidity & $48 \%$ & $48 \%$ & $48 \%$ & 0.94 & $48 \%$ & $48 \%$ & 0.87 \\
\hline In intervention arm & $88 \%$ & $83 \%$ & $88 \%$ & 0.09 & $87 \%$ & $88 \%$ & 0.73 \\
\hline APOL1 high risk & $13 \%$ & $15 \%$ & $13 \%$ & 0.43 & $14 \%$ & $13 \%$ & 0.6 \\
\hline Have a PCP & $95 \%$ & $94 \%$ & $95 \%$ & 0.46 & $95 \%$ & $95 \%$ & 1.00 \\
\hline 1st time in research & $78 \%$ & $85 \%$ & $77 \%$ & 0.07 & $85 \%$ & $77 \%$ & 0.06 \\
\hline Anxiety & $22 \%$ & $21 \%$ & $22 \%$ & 0.92 & $21 \%$ & $22 \%$ & 0.76 \\
\hline
\end{tabular}

$B P$ blood pressure, $H S$ high school, $P C P$ primary care provider.

The "great party" signifies that once patients and providers joined GUARDD, we aimed to optimize their research experience, so they could benefit from and remain in the trial. We piloted and revised our strategies and addressed struggles with our genomics board, who suggested some of our most successful strategies, including intercepting patients at clinical visits to discuss the study or follow-up, and ensuring participants would not go more than 3 months without hearing from study staff. Patients met with the same recruiters, who got to know them, kept in touch with them, and would meet them at convenient times. Many patients do not have continuity in their health care and appreciated the opportunity to build rapport with study staff. For PCPs, we developed the results form and content of EHR alerts that they requested and checked in with them throughout the study to address concerns.

Loss to follow-up in GUARDD was low, and the demographic difference between those lost and those retained (male sex and younger age) have been described in other studies. Variables we hypothesized would make it difficult for patients to continue in the study, including poverty, depression, medical mistrust, and poorer self-rated health did not predict rates of follow-up. This may be due to the patient-centered approaches we employed to maximize retention, or because these variables are less significant than we hypothesized. A notable exception is that patients with lower health literacy were less likely to follow up despite having all study materials at a sixth grade reading level, administering all consents and surveys verbally, and having recruiters communicate with patients nearly exclusively verbally. We did not assess literacy of patients who declined, so we do not know if it impacted enrollment rates. However, literacy is important for medical decision-making. Other studies associate low health literacy with study attrition, ${ }^{34}$ lower comprehension of informed consent and procedures for study follow-up, and avoidance of health care due to prior challenges navigating it. ${ }^{35,36}$

Limits of EHR sampling warrant further discussion. Half the patients we identified this way were ineligible. EHRs are built for patient care, and repurposing them for research can be challenging, as EHR data is often inaccurate, incomplete, and can be biased. ${ }^{31}$ Though we used algorithms combining clinical data and diagnostic codes, nearly half of our ineligible patients stated they did not have hypertension or did have diabetes and chronic kidney disease (CKD) (exclusionary diseases). Some may not have known about, misunderstood, 
disagreed with, or been in denial about their diagnoses. Others may have denied a diagnosis to avoid the discomfort of having to decline participation. Future studies may improve the sensitivity of EHR identification of study participants through improved algorithms and natural language processing. ${ }^{37}$ It was difficult to use EHRs to identify AA patients, as race-ethnicity fields are commonly missing or inaccurate, even in settings that care mainly for minority patients. ${ }^{38,39}$ In GUARDD, over $10 \%$ of patients told us their listing as Black race was wrong. We were also unable to identify and thus contact AA patients who were among the one-third who met all other EHR criteria for eligibility, but had missing race-ethnicity data. Increasing regulations may improve the accuracy of these fields for both research and patient care. ${ }^{40}$

We successfully recruited and retained AA patients with many potential barriers to research participation into a clinical trial of chronic disease genomic risk, using a stakeholderengaged, patient-centered approach. Our analysis has limitations. We did not randomize patients to recruitment with and without robust engagement, or to different types of recruitment strategies, so we could not rigorously compare their relative impacts. We had limited information on decliners. We also do not have a category of patients we did not reach, in part because we contacted their next of kin or would intercept them at clinical visits, and in part because we finished enrollment before we reached the maximum 12 phone calls we would place per patient, so we could not analyze data on unreachable individuals. However, our approach required far more than sending emails and letters. Hands-on approaches may be necessary to engage diverse populations in research.

Through building trust and rapport with patients, we created a research community that includes patients and their PCPs, which may have engendered a sense of research citizenship. Many patients asked for information about other research opportunities and 100 joined an intensive metabolic study nearly a year after GUARDD study completion. Studies that attend to the needs and preferences and optimize the experiences of participants may have reach far beyond a single study, particularly in minority groups that are underrepresented in research.

\section{ACKNOWLEDGEMENTS}

This study is supported by National Human Genome Research Institute (NHGRI) (5U01HG007278, U01HG006380) and National Center for Advancing Translational Sciences (NCATS) (UL1TR000067). Neither NHGRI nor NCATS had any involvement in the study design, collection, analysis or interpretation of data, writing of this article, or decision to submit it for publication. The authors also thank the GUARDD team of academic, community, and clinical partners; study coordinators; staff at study sites; and their partners in the IGNITE Network, a consortium of genomic medicine pilot demonstration projects funded and guided by the NHGRI, for their valuable contributions to this project.

\section{DISCLOSURE}

The authors declare no conflicts of interest.
Publisher's note: Springer Nature remains neutral with regard to jurisdictional claims in published maps and institutional affiliations.

\section{REFERENCES}

1. Corbie-Smith G, Thomas SB, St George DM. Distrust, race, and research. Arch Intern Med. 2002;162:2458-2463.

2. Scharff DP, Mathews KJ, Jackson P, Hoffsuemmer J, Martin E, Edwards D. More than Tuskegee: understanding mistrust about research participation. J Health Care Poor Underserved. 2010;21:879-897.

3. Yancey AK, Ortega AN, Kumanyika SK. Effective recruitment and retention of minority research participants. Annu Rev Public Health. 2006;27:1-28.

4. McDonald JA, Vadaparampil S, Bowen D, Magwood G, Obeid JS, Jefferson $\mathrm{M}$, et al. Intentions to donate to a biobank in a national sample of African Americans. Public Health Genomics. 2014;17:173-182.

5. Ngui EM, Warner TD, Roberts LW. Perceptions of African-American health professionals and community members on participation of children and pregnant women in genetic research. Public Health Genomics. 2014;17:23-32.

6. Buseh AG, Stevens PE, Millon-Underwood S, Townsend L, Kelber ST. Community leaders' perspectives on engaging African Americans in biobanks and other human genetics initiatives. J Community Genet. 2013;4:483-494.

7. Moore EG, Roche M, Rini C, Corty EW, Girnary Z, O'Daniel JM, Lin FC, Corbie-Smith G, Evans JP, Henderson GE, Berg JS. Examining the cascade of participant attrition in a genomic medicine research study: barriers and facilitators to achieving diversity. Public Health Genomics. 2017; 20:332-342

8. Fullerton SM, Knerr S, Burke W. Finding a place for genomics in health disparities research. Public Health Genomics. 2012;15:156-163.

9. Johnson VA, Powell-Young YM, Torres ER, Spruill IJ. A systematic review of strategies that increase the recruitment and retention of African American adults in genetic and genomic studies. ABNF J. 2011;22:84-88.

10. Bussey-Jones J, Garrett J, Henderson G, Moloney M, Blumenthal C, Corbie-Smith $\mathrm{G}$. The role of race and trust in tissue/blood donation for genetic research. Genet Med. 2010;12:116-121.

11. Streicher SA, Sanderson SC, Jabs EW, et al. Reasons for participating and genetic information needs among racially and ethnically diverse biobank participants: a focus group study. J Community Genet. 2011;2:153-163.

12. Isler MR, Sutton K, Cadigan JR, Corbie-Smith G. Community perceptions of genomic research: implications for addressing health disparities. N C Med J. 2013;74:470-476.

13. Furr AL. Perceptions of genetics research as harmful to society: differences among samples of African- Americans and EuropeanAmericans. Genet Test. 2004;6:25-30.

14. Johns TS, Estrella MM, Crews DC, et al. Neighborhood socioeconomic status, race, and mortality in young adult dialysis patients. J Am Soc Nephrol. 2014;25:2649-2657.

15. Hensley Alford S, McBride CM, Reid RJ, Larson EB, Baxevanis AD, Brody LC. Participation in genetic testing research varies by social group. Public Health Genomics. 2011;14:85-93.

16. Sisk JE, Horowitz CR, Wang JJ, Hebert PL, McLaughlin MA, Tuzzio L. The success of recruiting minorities, women, and elderly into a randomized controlled effectiveness trial. Mt Sinai J Med. 2008;75:37-43.

17. Horowitz CR, Brenner BL, Lachapelle S, Amara DA, Arniella G. Effective recruitment and enrollment through community-led strategies. Am J Prev Med. 2009;37:S195-200.

18. Kronish I, Goldfinger JZ, Negron R, Fei K, Arniella G, Horowitz CR. The effect of peer education on stroke prevention: the Prevent Recurrence of All Inner-City Strokes through Education (PRAISE) randomized controlled trial. Stroke. 2014;45:3330-3336.

19. Weitzel KW, Alexander M, Bernhardt BA, et al. The IGNITE Network: a model for genomic medicine implementation and research. BMC Med Genomics. 2016;9:1.

20. Parsa A, Kao WH, Xie D, et al. APOL1 risk variants, race, and progression of chronic kidney disease. N Engl J Med. 2013;369:2183-2196.

21. Horowitz C, Abul-Husn N, Ellis $S$, et al. Determining the effects and challenges of incorporating genetic testing into primary care management of hypertensive patients with African ancestry. Contemp Clin Trials. 2016;47:101-108.

22. Kaplan B, Caddle-Steele C, Chisholm G, et al. A culture of understanding: reflections and suggestions from a genomics 
research community board. Prog Community Health Partnersh. 2017; 11:161-165

23. Horowitz $C R$, Ferryman $K$, Negron $R$, et al. Race, genomics and chronic disease: what patients with African ancestry have to say. J Health Care Poor Underserved. 2017;28:248-260.

24. McDonald JA, Barg FK, Weathers B, et al. Understanding participation by African Americans in cancer genetics research. J Natl Med Assoc. 2012;104:324-330.

25. Harris PA, Taylor R, Thielke R, Payne J, Gonzalez N, Conde JG. Research electronic data capture (REDCap) - a metadata-driven methodology and workflow process for providing translational research informatics support. J Biomed Inform. 2009;42:377-381.

26. Huang GD, Bull J, Johnston McKee K, Mahon E, Harper B, Roberts JN, CTTI Recruitment Project Team. Clinical trials recruitment planning: a proposed framework from the Clinical Trials Transformation Initiative. Contemp Clin Trials. 2018;66:74-79.

27. Bates BR, Lynch JA, Bevan JL, Condit CM. Warranted concerns, warranted outlooks: a focus group study of public understandings of genetic research. Soc Sci Med. 2005;60:331-344.

28. Thompson HS, Valdimarsdottir HB, Jandorf L, Redd W. Perceived disadvantages and concerns about abuses of genetic testing for cancer risk: differences across African American, Latina and Caucasian women. Patient Educ Couns. 2003;51:217-227.

29. Laskey SL, Williams J, Pierre-Louis J, O'Riordan M, Matthews A, Robin NH. Attitudes of African American premedical students toward genetic testing and screening. Genet Med. 2003;5:49-54.

30. Sofolahan-Oladeinde $Y$, Mullins $C D$, Baquet $C$. Using community-based participatory research in patient-centered outcomes research to address health disparities in under-represented communities. J Comp Eff Res. 2015;4:515-523.

31. Fouad $M N$, Partridge $E$, Green $B L$, et al. Minority recruitment in clinical trials: a conference at Tuskegee, researchers and the community. Ann Epidemiol. 2000;10 8 Suppl:S35-40.

32. Simon V. Wanted: women in clinical trials. Science. 2005;308:1517.

33. Ali N, Lifford KJ, Carter B, et al. Barriers to uptake among high-risk individuals declining participation in lung cancer screening: a mixed methods analysis of the UK Lung Cancer Screening (UKLS) trial. BMJ Open. 2015;5:e008254.

34. Hardman R, Lawn S, Tsourtos G. Pain self-management: easier said than done? Factors associated with early dropout from pain self-management in a rural primary care population. Pain Med. 2018;0:1-11.

35. Brabers $A E$, Rademakers JJ, Groenewegen PP, van Dijk L, de Jong JD. What role does health literacy play in patients' involvement in medical decision-making? PLoS ONE. 2017;12:e0173316.

36. Leak C, Goggins K, Schildcrout JS, et al. Effect of health literacy on research follow-up. J Health Commun. 2015;20:83-91.

37. Pathak J, Kho AN, Denny JC. Electronic health records-driven phenotyping: challenges, recent advances, and perspectives. JAMIA. 2013;20:206-211.

38. Lee SJ, Grobe JE, Tiro JA. Assessing race and ethnicity data quality across cancer registries and EMRs in two hospitals. JAMIA. 2016;23:627-634.

39. Klinger EV, Carlini SV, Gonzalez I, et al. Accuracy of race, ethnicity, and language preference in an electronic health record. J Gen Intern Med. 2015;30:719-723.

40. Chin MH. Using patient race, ethnicity, and language data to achieve health equity. J Gen Intern Med. 2015;30:703-705. 